\title{
Forecasting the Consumer Price Index (CPI) of Ecuador: A Comparative Study of Predictive Models
}

\author{
Juan Riofrío ${ }^{\mathrm{a}, 1}$, Oscar Chang ${ }^{\mathrm{a}, 2}$, E. J. Revelo-Fuelagán ${ }^{\mathrm{b}}$, Diego H. Peluffo-Ordóñez ${ }^{\mathrm{a}, 3}$ \\ ${ }^{a}$ Department of Computational Sciences, Yachay Tech University, Urcuquí, 100650, Ecuador

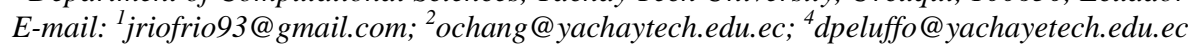 \\ ${ }^{b}$ Department of Electronic Engineering, Universidad de Nariño, Nariño, 52001, Colombia \\ E-mail: javierrevelof@udenar.edu.co
}

\begin{abstract}
The Consumer Price Index (CPI) is one of the most important economic indicators for countries' characterization and is typically considered an official measure of inflation. The CPI considers the monthly price variation of a determined set of goods and services in a specific region, and it is key in the economic and social planning of a given country, hence the great importance of CPI forecasting. In this paper, we outline a comparative study of state-of-the-art predictive models over an Ecuadorian CPI dataset with 174 monthly registers, from 2005 to 2019 . This small available dataset makes forecasting a challenging time-series-prediction task. Another difficulty is last year's trend variation, which since mid-2016, has changed from an upward average of 3.5 points to a stable trend of \pm 0.8 points. This paper explores the performance of relevant predictive models when tackling the Ecuadorian CPI forecasting problem accurately for the next 12 months. For this, a comparative study considering a variety of predictive models is carried out, including the Neural networks approach using a Sequential Model with Long Short-Term Memory layers machine learning using Support Vector Regression, as well as classical approaches like SARIMA and Exponential Smoothing. We also consider big corporations' tools like Facebook Prophet. As a result, the paper presents the best predictive models, and parameters found, along with Ecuador's CPI forecasting for the next 12 months (part of 2020). This information could be used for decisionmaking in several important topics related to social and economic activities.
\end{abstract}

Keywords - Consumer Price Index (CPI); Ecuador; predictive models; forecasting.

\section{INTRODUCTION}

Since Ecuador adopted dollarization in 2000, its economy has shown a persistent and robust development after a significant economic crisis that resulted in an annual inflation rate of $91 \%$ that year. The following years served to stabilize the country and its economy, as inflation rates began to decrease due to recession and the non-appearance of devaluation [1]. In the last years, Ecuador has had stable and low inflation, closing at $0.27 \%$ in 2018 and showing a recovery in the economic life of the country [2]. In Ecuador, as in most states, inflation is measured through the changes in the Consumer Price Index (CPI) between two periods.

The CPI is a monthly record that allows measuring throughout the time the variation of final consumption prices of a determined set of goods and services by households living in a specific urban area. In this sense, it is advisable to systematically change the CPI base year and update the goods and services of the market basket and its weights to let the index adjust to market evolution and changes in the population consumption. In Ecuador, six CPI base year changes have occurred. This work was developed with the last change, in 2014 [3]. In this update (Base: $2014=100$ ), the CPI goods and services basket consisted of 359 products divided into 12 groups and then into 43 subgroups. Roughly 25,350 product prices increase monthly in 9 cities in Ecuador. This change of the base year was based on the results of the National Survey of Income and Expenditure of Urban and Rural Households (ENIGHUR by its acronym in Spanish) conducted in 2011-2012.

The dataset analyzed in this work is the General CPI of Ecuador from 2005 to 2019, which contains 174 samples and the trend. Its small size makes it challenging to learn patterns since it is prone to over-fitting and holds few data to test results. The dataset shows a clearly growing trend varying an average of 3.5 points each year until 2016, and a stable trend in the same range $( \pm 0.8$ points) from then on. The data were taken from the Ecuadorian National Institute of Statistics and Censuses (INEC by its acronym in Spanish).

CPI is a key variable for a country's macroeconomic analysis, and it can be helpful when performing several studies. Some clear examples are its use in determining the 
cost of living in a city, inflation, or the minimum wage of a country. It can also be used to predict gold price returns as proposed by Sharma [4] or to forecast regional socioeconomic development [5]. Due to its importance, this study attempts to forecast the monthly values of CPI for the next year (July 2019 - June 2020) using 5 different time series predictive models. First, a machine learning approach was selected, represented by Support Vector Regression (SVR), using different kernels. Second, an Artificial Neural Network (ANN) approach using Long Short-Term Memory (LSTM) Layers was selected. The third predictive model used was the Seasonal Autoregressive Integrated Moving Average (SARIMA), a classical approach used in statistics and econometrics. The next predictive model used was the Exponential Smoothing model. Finally, a predictive method designed by Facebook called Prophet was used as well. All the implementations were done in Python programming language.

The remaining of this paper is structured as follows: Section II describes the dataset used and overviews the predictive models considered as well as the experimental methodology proposed. Section III gathers the results and discussion. Finally, the concluding remarks are drawn in section IV.

\section{MATERIALS AND METHOD}

\section{A. Dataset}

The "Consumer Price Index of Ecuador" dataset is a time series composed of 174 samples. Each one has been taken monthly from January 2005 to June 2019. The dataset is shown in Fig. 1.

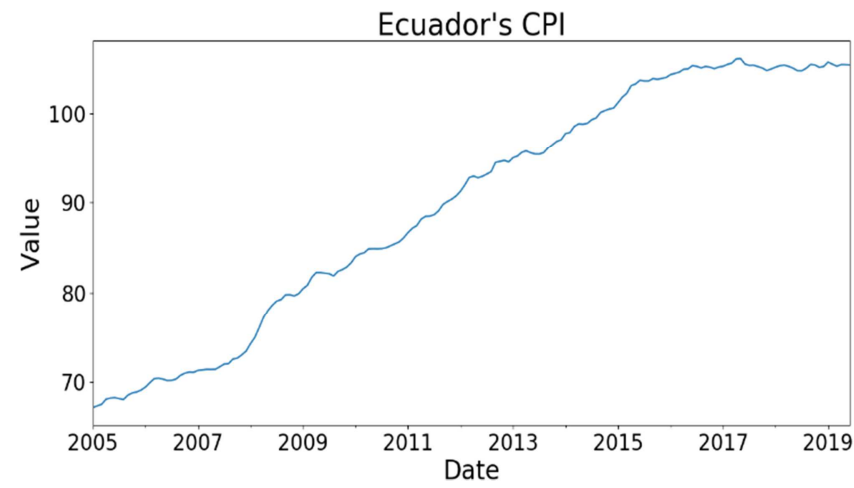

Fig. 1: 2005-20019 Ecuador's CPI time series plot

The only column of the dataset contains the CPI value for each month. Therefore, it is a vector of dimension $174 \times 1$. The dataset, together with the methodology used to calculate the monthly value of CPI, can be found in the official governmental website [6]. Table 1 presents the dataset description.

TABLE I

ECUADOR's CPI DATASET DESCRIPTION

\begin{tabular}{|c|c|c|c|c|}
\hline Count & Mean & Std. Deviation & Min & Max \\
\hline 174 & 89.957184 & 13.306077 & 67.12 & 106.17 \\
\hline
\end{tabular}

Figures 2, 3 and 4 show the seasonal decomposition of the time series.



Fig. 2: Trend attribute of Ecuador's CPI time series additive seasonal decomposition.

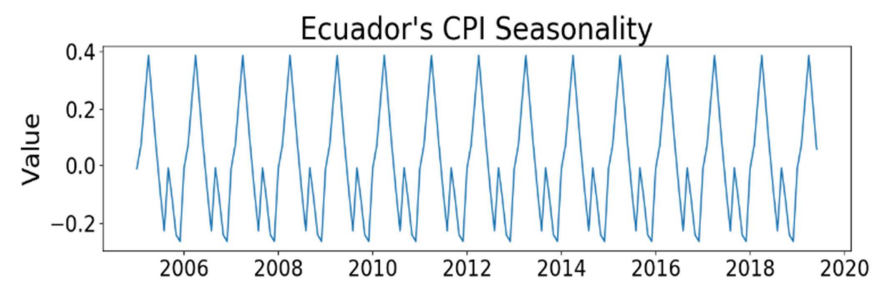

Fig. 3: Seasonality attribute of Ecuador's CPI time series additive seasonal decomposition. Frequency $=12$.

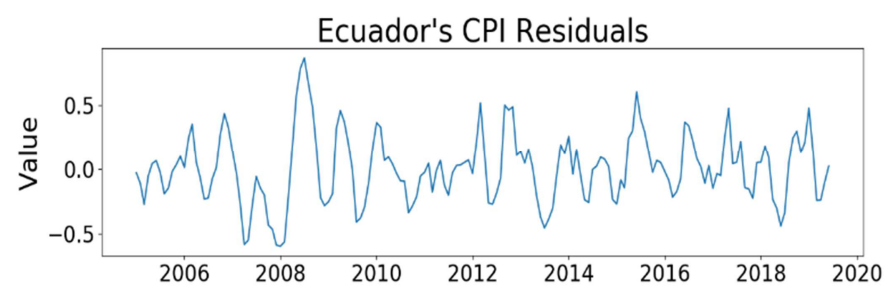

Fig. 4: Residuals attribute of Ecuador's CPI time series additive seasonal decomposition.

\section{B. Predictive Models}

The Ecuadorian CPI dataset was used to train and evaluate five different predictive models.

1) Support Vector Regression (SVR) [7]: This regression technique uses the same principle of the Support Vector Machine for classification, except for some differences so that it can be applied to continuous values. The idea is to consider a margin distance epsilon. So, all the samples are in a band around the selected hyperplane that best suits our data. Their distance from the hyperplane is smaller than epsilon. When defining the hyperplane, only examples that are more distant from our hyperplane than epsilon are taken. In SVR those samples will be considered as support vectors. SVR uses kernels to map the input into a higher dimensional space where we can find the hyperplane that best suits our data. In this work, we use Radial Basis Function (RBF) and Polynomial kernels for the SVR model.

2) Long Short-Term Memory (LSTM) Neural Network [8] [9]: LSTM is a recurrent neural network architecture developed by Sepp Hochreiter and Jürgen Schmidhuberin in the mid-'90s in response to the problems presented by recurrent neural networks. Recurrent neural networks need to monitor states, which is computationally expensive, and they have issues like the vanishing gradient and the exploding gradient. LSTM unit is an approach commonly used to deal with these issues.

LSTM unit reflects computer memory that works in conjunction with a recurrent network. It is utilized to maintain, update, and regulate the states of the network model outside of its typical execution flow. It consists of 
four main elements: the memory cell and three logistic gates. The memory cell holds information, and the logistic gates specify the flow of data inside the LSTM. The input gate writes data into the memory cell; the output gate is in charge of reading data from the information cell and sending it back to the recurrent network, whereas the forget gate decides whether to keep or delete data from the information cell. These gates have similarities with the neurons from a neural network, as they are multiplicative analog sigmoid-activated nodes. By manipulating these gates, a recurrent network can remember what it needs and forget what is no longer useful. The idea is to manipulate values through the gates to solve the vanishing gradient and exploding gradient problems. The gates let the network forget the states that are not required anymore, thus reducing the model's computational load radically. LSTM let recurrent networks keep learning over 1000-time steps by maintaining a constant error.

3) Seasonal Auto-Regressive Integrated Moving Average (SARIMA) [10]: Auto-Regressive Integrated Moving Average (ARIMA) is a statistical model that finds patterns from existing data to predict future values. Therefore, future predictions are described by past data and not by independent variables. It is one of the most used methods to forecast univariate time series data. It has three hyperparameters to specify $(\mathrm{p}, \mathrm{d}, \mathrm{q})$, where ' $\mathrm{p}$ ' is the autoregression term seen as the lags of the previous value, ' $d$ ' is the integral (differencing) term for making time series stationary. The moving average term ' $q$ ' is a past error (multiplied by a coefficient).

SARIMA is an extension of the ARIMA model, with the difference that SARIMA supports time series with a seasonal component. To make this possible, it adds 4 new hyperparameters (sp,sd,sq, s), where three of them are similar to ARIMA hyperparameters (p,d,q). Still, they implicate back-shifts of the seasonal period. The last hyperparameter added ('s') is the period of the seasonality where 12 is the monthly data, 4 is the quarterly data, 0 is no seasonal data, etc.

4) Exponential Smoothing [11]: It is a predictive model for univariate data that can support data with a seasonal component or systematic trend by adding some variations. Exponential smoothing uses the linear combination of past values to forecast future values. The difference with moving average is that exponential smoothing sets exponentially decreasing weights as the observation gets older, which means that more recent values are associated with higher weights, so they have more influence in the predicted value. Precisely, it weighs past observations with a geometrically declining ratio.

5) Facebook Prophet [12]: It is a software designed by Facebook's Core Data Science team. The so-named Prophet approach is a time series forecasting method that is easy to use and tune. It is based on an additive model where nonlinear trends fit with yearly, weekly, and daily seasonality, plus holiday effects. Prophet properly handles missing data, changes in trend, and outliers. This predictive model works best with data that have a strong seasonality and some seasons of historical data. Facebook uses this software in its application for its predictions, but because it is easy to use and tune, it is also being used by amateur data scientists. The Python library used for this model is fbprophet, to see the documentation of the procedure go to https://github.com/facebook/prophet.

\section{Metrics}

The mean absolute percentage error was used to compare the efficiency of the predictive. In SARIMA model, the AIC estimator was also used.

1) Mean absolute Percentage Error (MAPE) [13]: It is the average of the absolute percentage errors of the predictions. This measure is straightforward as it shows how significant prediction errors are compared to the values of the series in terms of percentage. The smaller the MAPE, the better the predictions. MAPE can be expressed as follows:

$$
M A P E=\frac{1}{n} \sum_{t=1}^{n} \frac{\left|Y_{t}-\bar{Y}_{t}\right|}{\left|Y_{t}\right|}
$$

where $Y_{t}$ is the actual value at time $t, \bar{Y}_{t}$ is the predicted value at the time $t$ and $n$ is the number of observations.

2) Akaike information criterion (AIC) [14]: AIC approximates the relative quantity of information lost by a selected model; thus, if the model loses less information, it is a higher quality model. By adding parameters to the model, we get better results, but we are also trading off against overfitting and losing information about the inherent pattern. AIC represents a trade-off between an added number of parameters and the error that we are considering by adding said parameters. The model that fits better will have the lowest AIC.

\section{Experimental Methodology:}

For all the experiments (see the next section), the predictive models were trained using all the data except for the last 12 months, as seen in Fig. 5. The last 12 months (test dataset) presented in Fig. 5 were used for testing the models. All experiments were run in Python using already existing modules of predictive models. A grid search was performed to look for the best hyperparameters for each kind of predictive model, comparing the possible configurations and selecting the one with the lowest MAPE. This is done for each predictive model, and in the end, the forecasting of the next 12 months is performed to compare the best configuration of each predictive model against each other. Fig. 6 summarizes graphically the methodology used in this research.

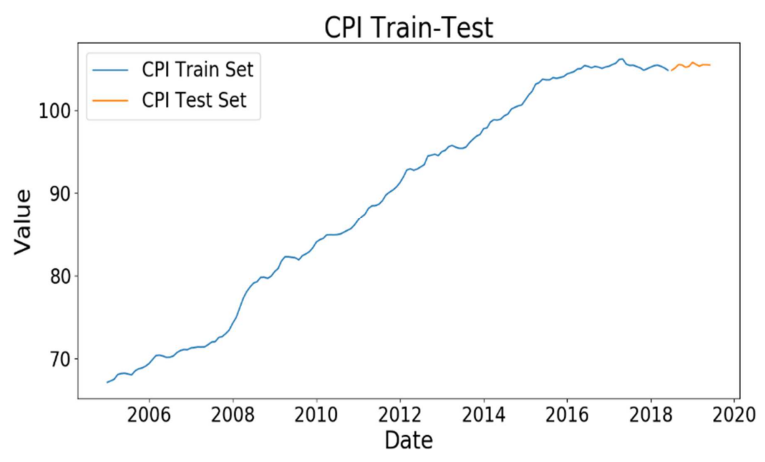

Fig. 5: CPI train \& test datasets plot 


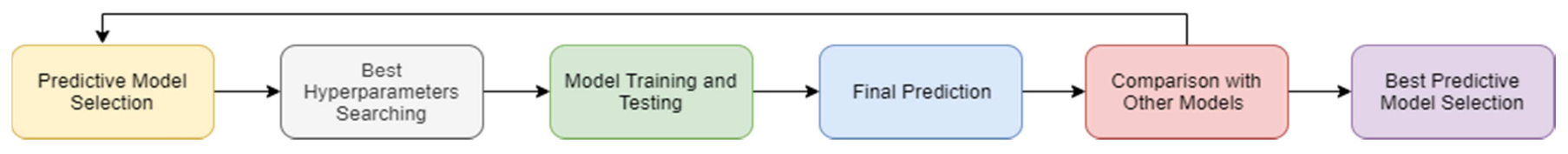

Fig. 6: Block diagram of the proposed methodology.

\section{RESULTS AND DISCUSSION}

\section{A. SVR Results}

For the SVR model, two different kernels were used: RBF and polynomial. For each kernel, a grid search was performed with a range of values to look for the best hyperparameter combination. The implementation was made with the help of the sci-kit-learn library and the SVR method. The best configurations were the SVR using RBF kernel with an epsilon value of 1 , a penalty value of the error of 10, a kernel coefficient of 0.0001 ; and SVR using the polynomial kernel of degree 8 and an independent term value of 9 , with an epsilon value of 0.1 , a penalty value of the error of 1 , and a kernel coefficient of 0.1 . After training our models, we proceeded to forecast the testing values and find the MAPE for each model. In the case of the RBF kernel, the MAPE was 0.00254, whereas the MAPE for the polynomial kernel was 0.00171 . The results of the SVR models are presented in Figures 7, 8, 9, 10, and 11.



Fig. 7: Comparison of SVR predictions for the test datasets.

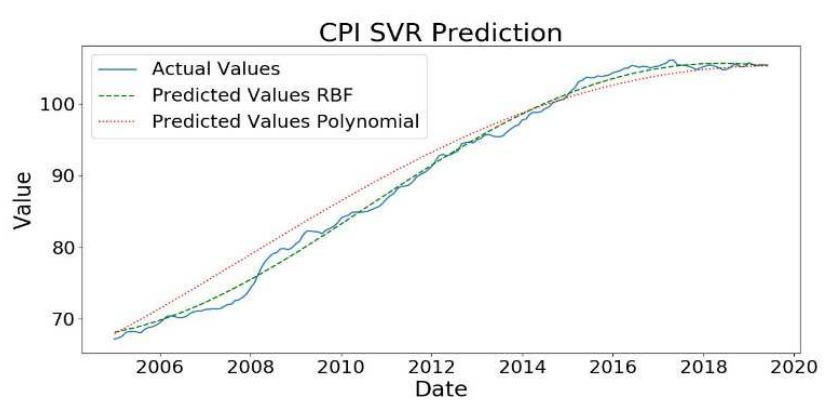

Fig. 8: SVR models fitting to the time series.



Fig. 9: CPI dataset and final predictions of SVR model using RBF kernel.

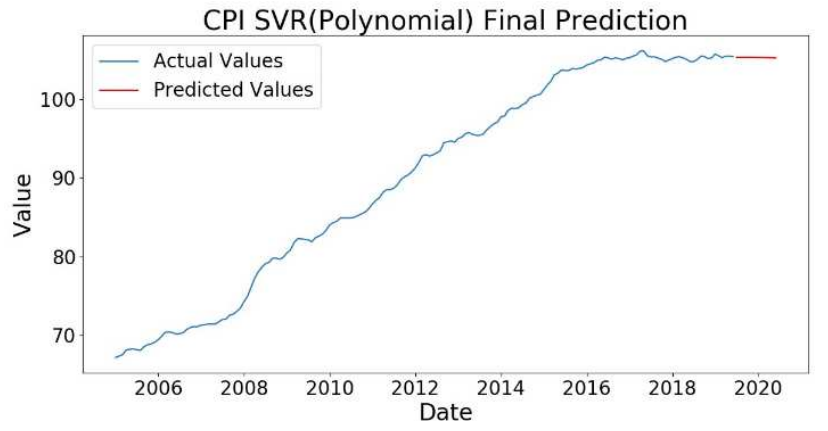

Fig. 10: CPI dataset and final predictions of the SVR model using the polynomial kernel.

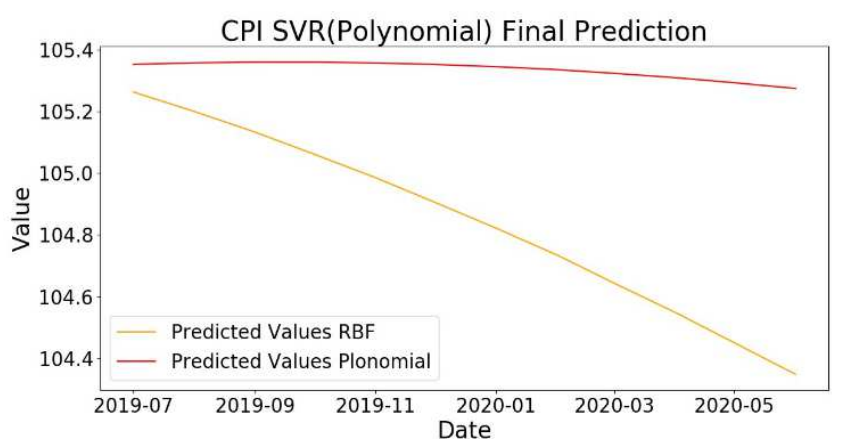

Fig. 11: Comparison of final predictions of SVR models

\section{B. LSTM Neural Network Results}

To use this predictive model, we first created an artificial neural network (ANN), which was done with Keras library running on top of TensorFlow. Instead of a grid search for the parameters, we tried different architectures varying the number of layers, as well as the number of units in the layers until obtaining the best possible MAPE. The selected ANN is a sequential model of layers, 3 LSTM layers, 3 Dropout layers, and one dense layer. The number of units for the LSTM layers was six and for the dense layer, the unit was 1; the network architecture is presented in Fig. 12.

\begin{tabular}{|c|c|c|}
\hline \multicolumn{3}{|l|}{ Model: "sequential" } \\
\hline Layer (type) & Output Shape & Param \# \\
\hline $\begin{array}{l}========== \\
1 \mathrm{stm} \text { (LSTM) }\end{array}$ & $\begin{array}{c}===========3 \\
\text { (None, 12, 6) }\end{array}$ & $\begin{aligned} &==== \\
& 192\end{aligned}$ \\
\hline dropout (Dropout) & (None, 12,6 ) & 0 \\
\hline 1stm_1 (LSTM) & (None, 12,6 ) & 312 \\
\hline dropout_1 (Dropout) & (None, 12,6 ) & 0 \\
\hline 1 stm_2 (LSTM) & (None, 12, 6) & 312 \\
\hline dropout_2 (Dropout) & (None, 12, 6) & $\theta$ \\
\hline 1stm_3 (LSTM) & (None, 6) & 312 \\
\hline dropout_3 (Dropout) & (None, 6) & 0 \\
\hline dense (Dense) & (None, 1) & 7 \\
\hline \multicolumn{3}{|l|}{$\begin{array}{l}\text { Total params: } 1,135 \\
\text { Trainable params: } 1 \text {, }\end{array}$} \\
\hline \multicolumn{3}{|c|}{ Non-trainable params: $\theta$} \\
\hline
\end{tabular}

Fig. 12: LSTM Neural Network architecture summary. 
After creating the model, the training and testing stages were done, and in this case, the obtained MAPE was 0.00173. Because the input in this model is different, it is essential to mention that forecast a value the selected model uses the past 12 values to predict the next one after training. So, to predict the first value, we used the past 12 months, added the prediction to the input set, and then used it to predict the next values. This method is better to use if you need to predict only one value into the future as it requires the previous values to predict. The results of the LSTM neural network are provided in Figures 13, 14, and 15.

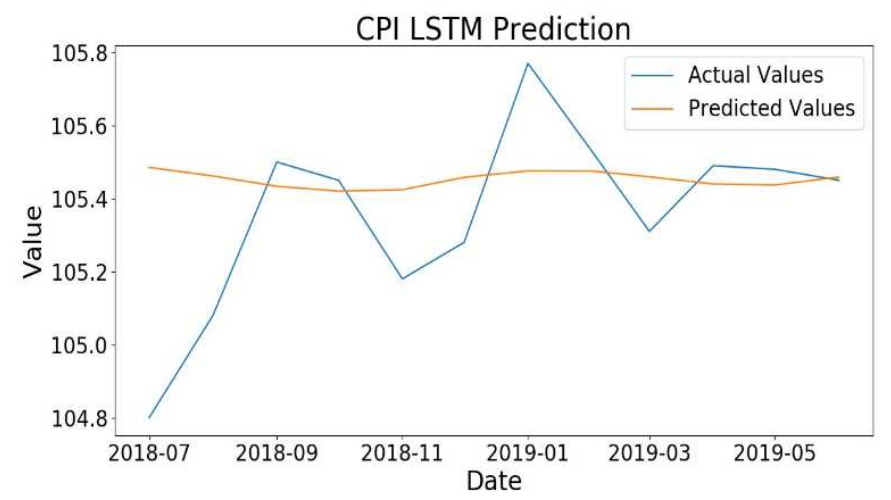

Fig. 13: LSTM model predictions for the test datasets

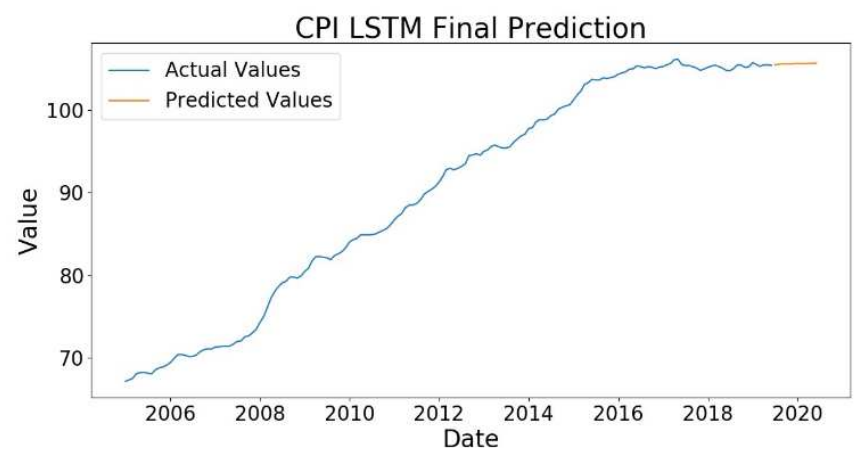

Fig. 14: CPI dataset and final predictions of LSTM model.

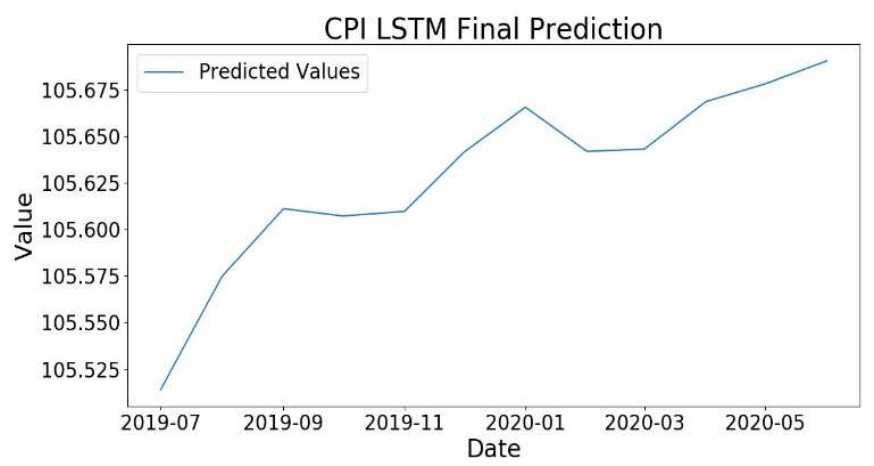

Fig. 15: Final predictions of the LSTM model.

\section{SARIMA Results}

In the SARIMA model, 2 different metrics were used to test the results of the hyperparameters search. First, the same approach as the other experiments was used, searching for tuning with the less MAPE. In the second approach, a search for the hyperparameters to get the best AIC was performed. We used the method SARIMAX from the library stats models to create and train our model. The best configuration to get the lowest MAPE for the SARIMA model was found with the following hyperparameters $(p=2, d=2, q=4),(s p$
$=1, \mathrm{sd}=0, \mathrm{sq}=1, \mathrm{~s}=0$ ). A MAPE of 0.00181 was obtained with this configuration. The best hyperparameter to get the best AIC was found with the help of the auto_arima function from the pmdarima library, while the best configuration found for the hyperparameters was $(\mathrm{p}=1, \mathrm{~d}=1, \mathrm{q}=1)$, (sp $=0, \mathrm{sd}=1, \mathrm{sq}=1, \mathrm{~s}=12$ ). After training and testing the results of the model a higher MAPE of 0.00550 was obtained, as expected. The results of the SARIMA models are presented in Figures 16,17, 18 and 19.

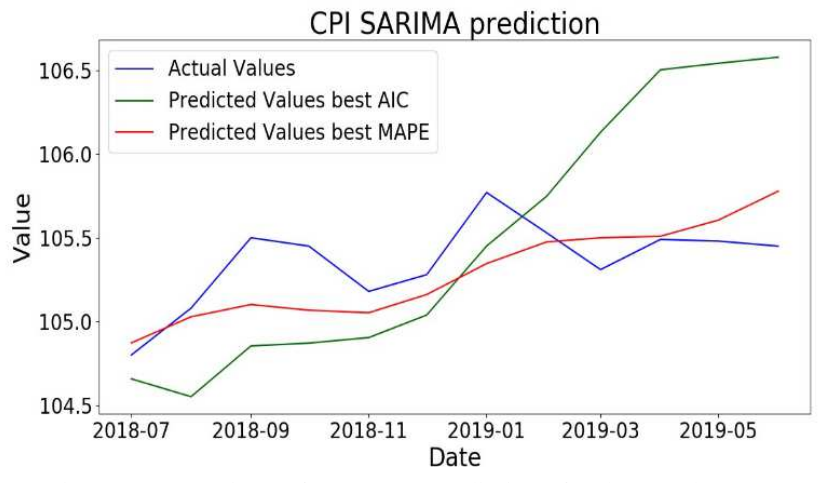

Fig. 16: Comparison of SARIMA predictions for the test datasets.



Fig. 17: CPI dataset and final predictions of SARIMA $(2,2,4)-(1,0,1,0)$ model.

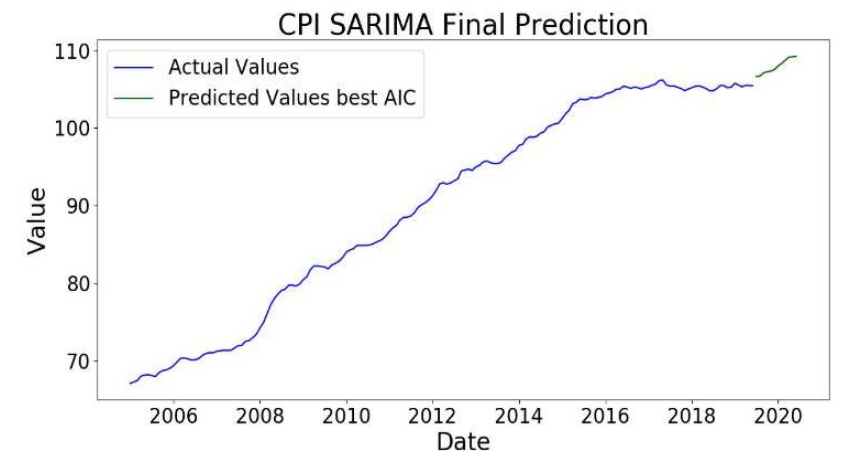

Fig. 18: CPI dataset and final predictions of SARIMA $(1,1,1)-(0,1,1,12)$ model.

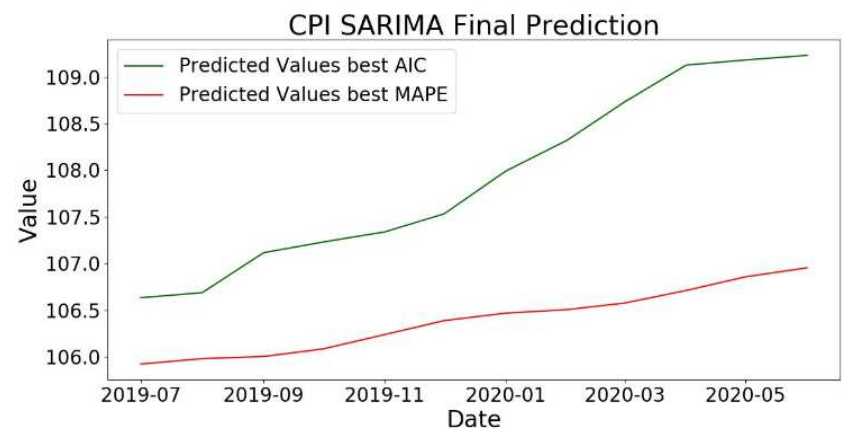

Fig. 19: Comparison of final predictions of SARIMA models. 


\section{Exponential Smoothing Results}

The model was created using the Exponential Smoothing method from the stats model library. The same procedure as before was applied, and a grid search was done to look for the best configuration to obtain the lowest MAPE. The best configuration found for the exponential smoothing method was a model using an additive type of trend without damping it, and a seasonal additive component of 12 periods per season. The predictions obtained with this configuration produced a MAPE of 0.00316 when compared to the test values. All the results of the exponential smoothing model are shown in Figures 20, 21 and 22.

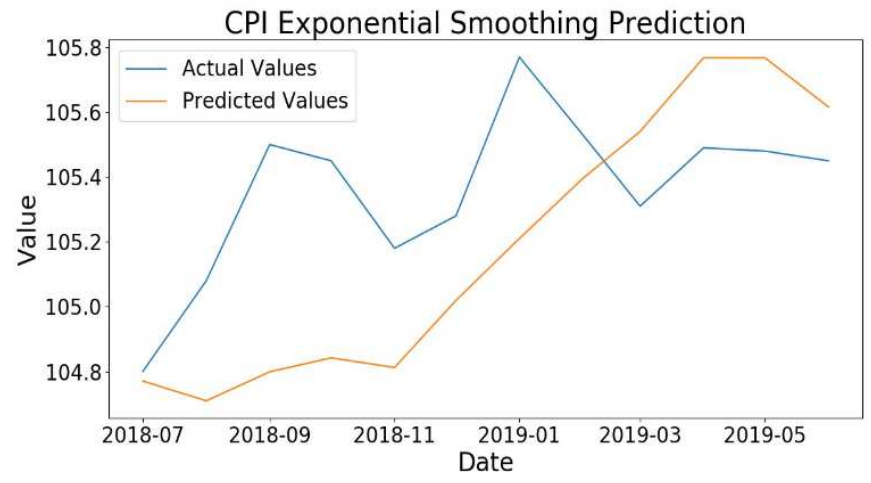

Fig. 20: Exponential Smoothing model predictions for test dataset.

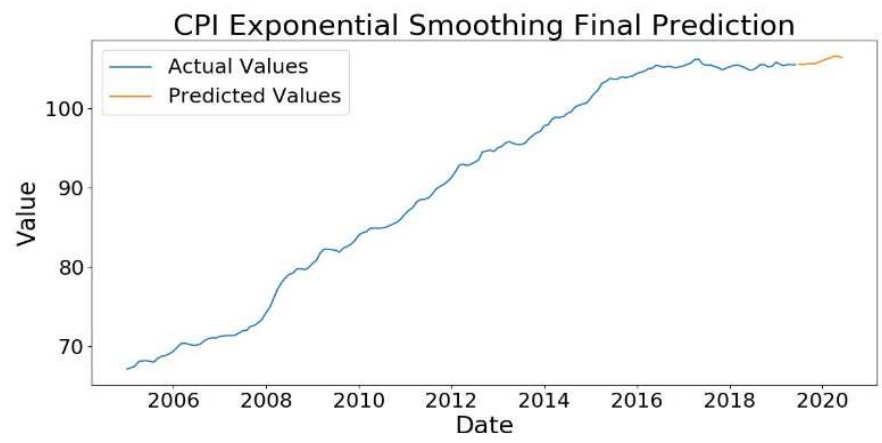

Fig. 21: CPI dataset and final predictions of Exponential Smoothing model.

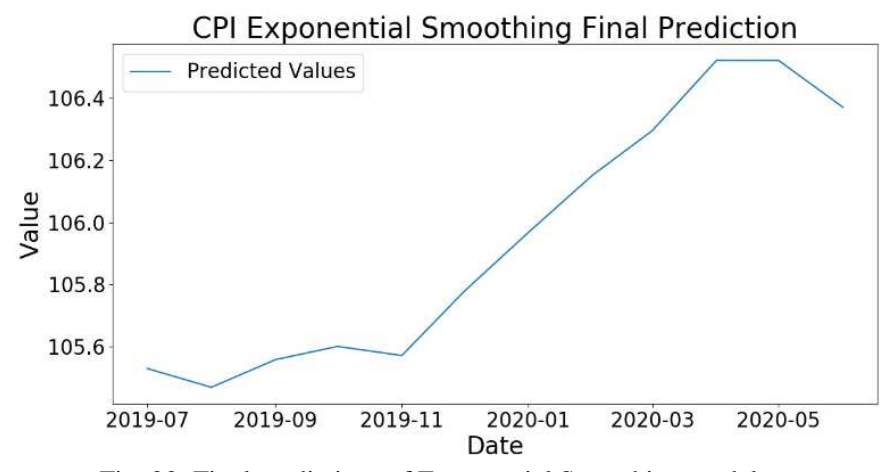

Fig. 22: Final predictions of Exponential Smoothing model.

\section{E. Prophet Results}

For the Prophet model, as mentioned before, we used the fbprophet library and the Prophet method. First, we performed the grid search to get the best configuration for the model and used MAPE again to evaluate the best model. The best configuration obtained from this step was the model with linear growth, with an interval width of 0.95 and yearly seasonality. The model trained with this configuration produces a MAPE of 0.00403 , higher than the other methods. The results produced by this model are presented in Figures 23, 24 and 25.

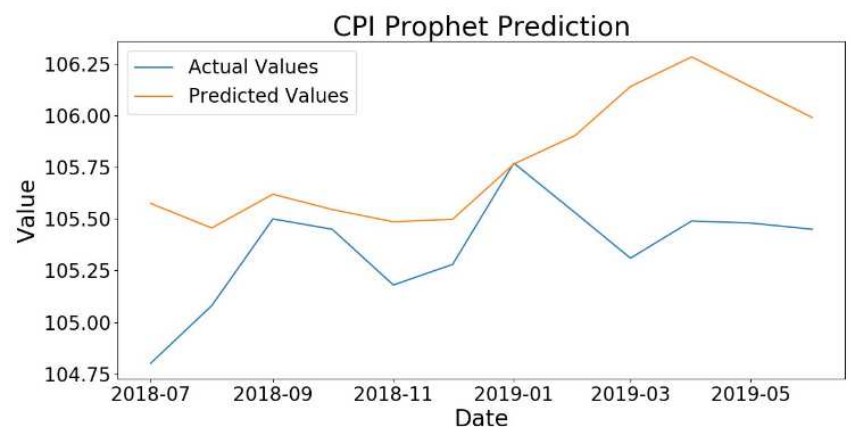

Fig. 23: Prophet model predictions for test dataset.

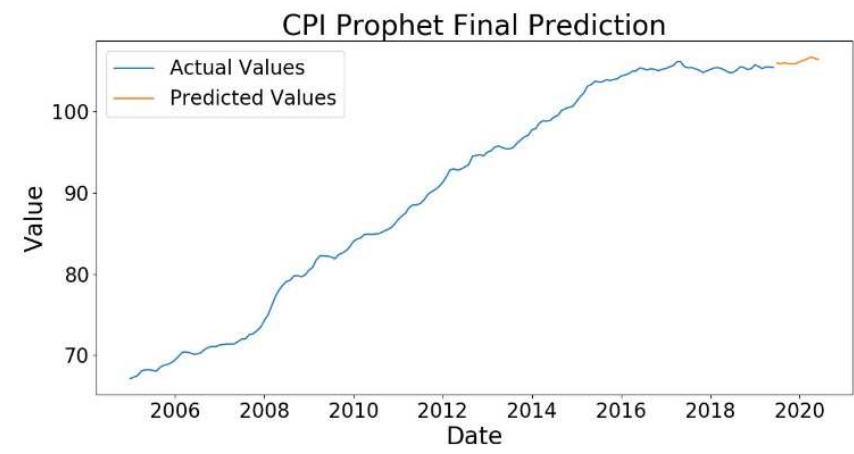

Fig. 24: CPI dataset and final predictions of Prophet model.

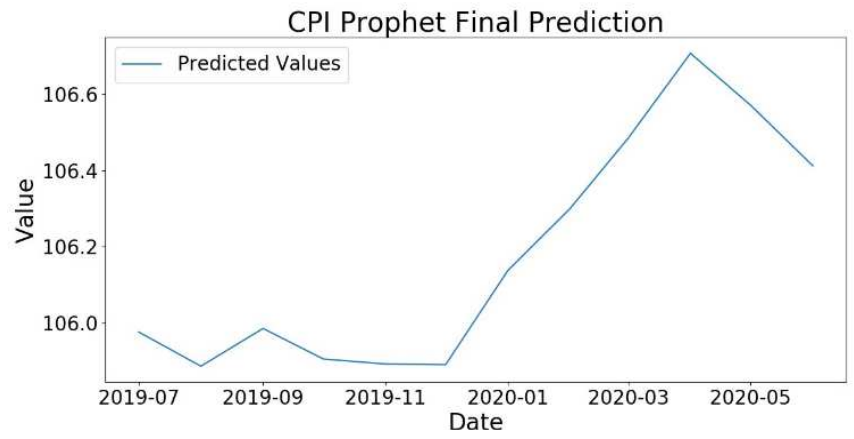

Fig. 25: Final predictions of Prophet model.

\section{F. Final Remarks}

Finally, Table 2 depicts the final predictions of all the models for the next year.

TABLE II

Monthly Final Predictions Of EACh Predictive Model

\begin{tabular}{|l|l|l|l|l|l|l|l|}
\hline Month & SVR Poly & SVR RBF & LSTM & $\begin{array}{l}\text { SARIMA } \\
\text { (MAPE) }\end{array}$ & $\begin{array}{c}\text { SARIMA } \\
\text { (AIC) }\end{array}$ & Exp. Smt & Prophet \\
\hline $2019-07$ & 105.3524 & 105.2632 & 105.5138 & 105.9237 & 106.6336 & 105.3726 & 105.9764 \\
\hline $2019-08$ & 105.3572 & 105.1999 & 105.5747 & 105.9816 & 106.6881 & 105.4020 & 105.8868 \\
\hline $2019-09$ & 105.3596 & 105.1325 & 105.6111 & 106.0048 & 107.1160 & 105.5901 & 105.9857 \\
\hline $2019-10$ & 105.3595 & 105.0609 & 105.6072 & 106.0860 & 107.2300 & 105.5512 & 105.9057 \\
\hline $2019-11$ & 105.3570 & 104.9853 & 105.6097 & 106.2392 & 107.3383 & 105.4663 & 105.8925 \\
\hline $2019-12$ & 105.3521 & 104.9057 & 105.6414 & 106.3885 & 107.5314 & 105.6079 & 105.8909 \\
\hline $2020-01$ & 105.3448 & 104.8221 & 105.6656 & 106.4690 & 107.9884 & 105.8691 & 106.1377 \\
\hline $2020-02$ & 105.3352 & 104.7347 & 105.6419 & 106.5060 & 108.3197 & 105.8896 & 106.2999 \\
\hline $2020-03$ & 105.3234 & 104.6436 & 105.6431 & 106.5768 & 108.7298 & 106.0110 & 106.4844 \\
\hline $2020-04$ & 105.3092 & 104.5487 & 105.6686 & 106.7112 & 109.1234 & 106.2835 & 106.7067 \\
\hline $2020-05$ & 105.2928 & 104.4503 & 105.6782 & 106.8578 & 109.1789 & 106.2133 & 106.5705 \\
\hline $2020-06$ & 105.2743 & 104.3483 & 105.6905 & 106.9542 & 109.2281 & 106.0051 & 106.4119 \\
\hline
\end{tabular}


Table 3 presents the MAPE obtained in each model when comparing the test dataset with the predictions for the same dates.

TABLE III

PREDICTIVE MODELS' MAPE

\begin{tabular}{|l|l|}
\hline Model & MAPE \\
\hline SVR Poly & 0.00171 \\
\hline SVR RBF & 0.00254 \\
\hline LSTM & 0.00173 \\
\hline SARIMA (MAPE) & 0.00181 \\
\hline SARIMA (AIC) & 0.00550 \\
\hline Exp. Smoothing & 0.00316 \\
\hline Prophet & 0.00403 \\
\hline
\end{tabular}

\section{IV.CONCLUSION}

The comparative study of the presented predictive models suggests that the Support Vector Regression (SVR) model using a polynomial kernel is the best element to predict the Ecuadorian CPI one year ahead in the future. The MAPE obtained with this solution was 0.00171. As seen in Figures 7 and 8 , it is good at predicting the trend rather than exact values. The model with the second-best MAPE was LSTM neural network, which obtained a mark of 0.00173. The predictions of this model, at least in its graphical representation, look realistic, with peaks and valleys, more like a real CPI should behave. In contrast, predictions with SVR using a polynomial (also RBF) tend to follow a smooth curve (Fig. 8). Considering that CPI can be used to estimate inflation, minimum wage, or the cost of living of a region, the results of this work can be used to calculate other relevant information for the Ecuadorian economy next year.

With the dataset used, other alternatives should be considered for future works. For example, the modification of a method or combination of two or more methods like the one proposed by Qin et al. to forecast China CPI based on EEMD and SVR method [15]. Another improvement could be extended for the grid to search through more possibilities of hyperparameters. Even better, optimize the search like the one proposed by $\mathrm{Cao}$ and $\mathrm{Wu}$ [16] for SVR forecasting, where the fruit fly optimization algorithm is used to search the best values of the hyperparameters automatically. New values are released every month, so the work of forecasting Ecuador's CPI is a rich source for further research.

\section{ACKNOWLEDGMENTS}

The authors acknowledge the research project "Desarrollo de una metodología de visualización interactiva y eficaz de información en Big Data" supported by Agreement No. 180 November 1st, 2016 by VIPRI from Universidad de Nariño. The authors thank the SDAS Research Group (www.sdasgroup.com), as well as Yachay Tech University (www.yachaytech.edu.ec) for their valuable support and guidance.

\section{REFERENCES}

[1] M. A. Terán Saltos, "Discursos sobre las causas de la inflación en una economía dolarizada." 2002.

[2] Banco Central del Ecuador(BCE)., "Ecuador: Reporte mensual de inflación.” 2018.

[3] Instituto Nacional de Estadisticas y Censos(INEC)., "Metodología del Índice de Precios al Consumidor (IPC) Base Anual: $2014=100$." 2015.

[4] S. S. Sharma, "Can consumer price index predict gold price returns?," Econ. Model., vol. 55, pp. 269-278, Jun. 2016.

[5] A. V Babkin, E. P. Karlina, and N. S. Epifanova, "Neural networks as a tool of forecasting of socioeconomic systems strategic development," Procedia-Social Behav. Sci., vol. 207, pp. 274-279, 2015.

[6] Instituto Nacional de estadistica y censos, "El índice de Precios al Consumidor (IPC)," 2020. [Online]. Available: https://www.ecuadorencifras.gob.ec/indice-de-precios-alconsumidor/.

[7] A. J. Smola and B. Schölkopf, "A tutorial on support vector regression," Statistics and Computing, vol. 14, no. 3. pp. 199-222, Aug-2004.

[8] S. Hochreiter and J. Schmidhuber, "Long Short-Term Memory," Neural Comput., vol. 9, no. 8, pp. 1735-1780, Nov. 1997.

[9] K. Greff, R. K. Srivastava, J. Koutn〉'lik, B. R. Steunebrink, and J. Schmidhuber, "LSTM: A search space odyssey," IEEE Trans. neural networks Learn. Syst., vol. 28, no. 10, pp. 2222-2232, 2016.

[10] G. E. P. Box and G. M. Jenkins, Time series analysis forecasting and control. San Francisco Holden-Day, 1970.

[11] R. G. Brown and R. F. Meyer, "The Fundamental Theorem of Exponential Smoothing," Oper. Res., vol. 9, no. 5, pp. 673-685, Oct. 1961.

[12] S. J. Taylor and B. Letham, "Forecasting at scale," Am. Stat., vol. 72, no. 1, pp. 37-45, 2018.

[13] A. De Myttenaere, B. Golden, B. Le Grand, and F. Rossi, "Mean Absolute Percentage Error for regression models," May 2016.

[14] S. Hu, "Akaike information criterion," Cent. Res. Sci. Comput., vol. 93, 2007.

[15] X. Qin, M. Sun, X. Dong, and Y. Zhang, "Forecasting of China Consumer Price Index Based on EEMD and SVR Method," in Proceedings - 2nd International Conference on Data Science and Business Analytics, ICDSBA 2018, 2018, pp. 329-333.

[16] G. Cao and L. Wu, "Support vector regression with fruit fly optimization algorithm for seasonal electricity consumption forecasting," Energy, vol. 115, pp. 734-745, Nov. 2016. 